PROFESIONALES Y HERRAMIENTAS PARA EL DESARROLLO LOCAL Y SUS SINERGIAS TERRITORIALES. EVALUACIÓN Y PROPUESTAS DE FUTURO IX Coloquio Nacional de Desarrollo Local del GTDL-AGE 

ANTONIO MARTÍNEZ PUCHE, XAVIER AMAT MONTESINOS, ISABEL SANCHO CARBONELL y DANIEL SANCHIZ CASTAÑO (EDS.)

\section{PROFESIONALES Y HERRAMIENTAS PARA EL DESARROLLO LOCAL Y SUS SINERGIAS TERRITORIALES. EVALUACIÓN Y PROPUESTAS DE FUTURO}

IX Coloquio Nacional de Desarrollo Local del GTDL-AGE

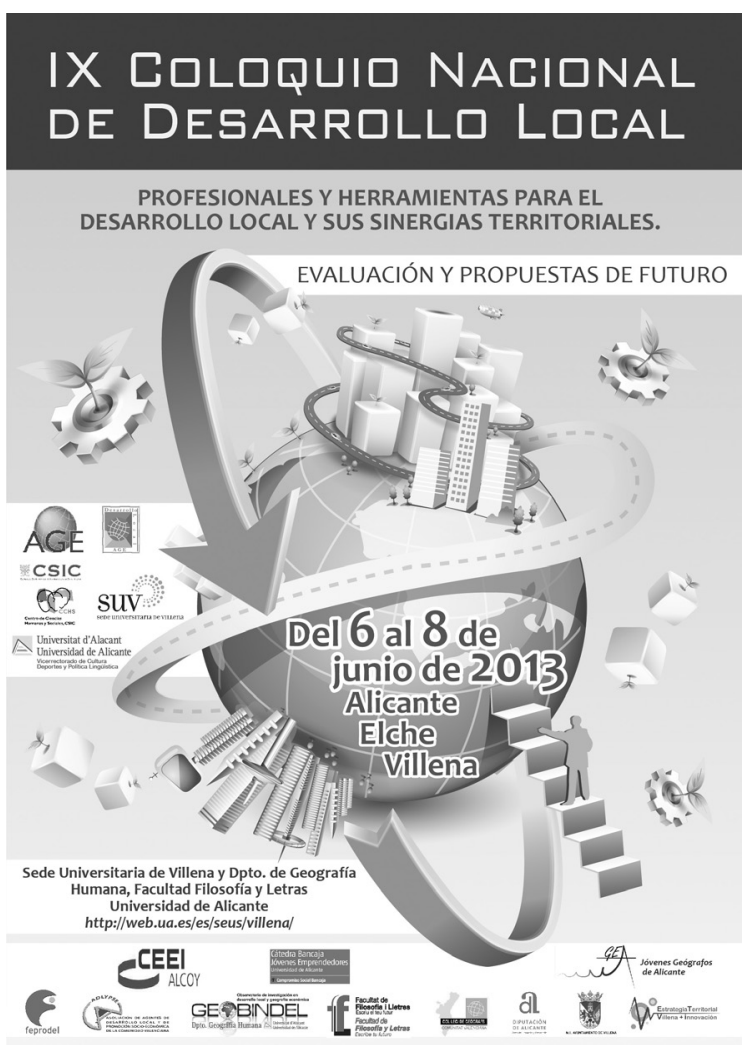


Este libro ha sido examinado y valorado por evaluadores ajenos a la Universidad de Alicante, con el fin de garantizar la calidad científica del mismo.

Publicacions de la Universitat d'Alacant

03690 Sant Vicent del Raspeig

Publicaciones@ua.es

http://publicaciones.ua.es

Telèfon: 965903480

(C) Antonio Martínez Puche, Xavier Amat Montesinos,

Isabel Sancho Carbonell y Daniel Sanchiz Castaño (eds.), 2016

(C) d'aquesta edició: Universitat d'Alacant

ISBN: 978-84-16724-00-0

Dipòsit legal: A 92-2016

Disseny de coberta: candela ink

Composició: Página Maestra (Miguel Ángel Sánchez Hernández)

Impressió i enquadernació: Guada Impresores

\section{unte \\ Unión de Editoriales
Universitarias Españolas \\ WWW.une.es
WWA}

Esta editorial es miembro de la UNE, cosa que garantiza la difusión y comercialización nacional y internacional de sus publicaciones.

Reservados todos los derechos. Cualquier forma de reproducción, distribución, comunicación pública o transformación de esta obra sólo puede ser realizada con la autorización de sus titulares, salvo excepción prevista por la ley. Diríjase a CEDRO (Centro Español de Derechos Repográficos, www.cedro.org) si necesita fotocopias o escanear algún fragmento de esta obra. 


\title{
LA GESTIÓN LOCAL DE LA SEGURIDAD CIUDADANA COMO HERRAMIENTA DE REVITALIZACIÓN Y DESARROLLO PARA LA COMUNIDAD
}

\author{
Shaila Villar García \\ Alumna Master Desarrollo Local e Innovación Territorial \\ shaila.vg@gmail.com
}

RESUMEN

La seguridad es una de las cuestiones sociales que más preocupan a los ciudadanos de cualquier territorio, y es elemento fundamental de desarrollo local. El concepto de seguridad ha estado enlazado control social/represión, pero esta idea ha ido evolucionando hacia la prevención de posibles conflictos.

Superando la relación tradicional " $\mathrm{n}^{\circ}$ de agentes= más seguridad", analizaremos, junto a las personas que habitan un territorio ¿Qué elementos son los que crean esa inseguridad? Y ¿Podemos crear herramientas que mejoren las condiciones de desarrollo de una comunidad?

Palabras clave: Seguridad ciudadana, Desarrollo local, prevención situacional, comunidad.

\section{SECURITY'S LOCAL MANAGEMENT AS A TOOL OF REVIVAL AND COMMUNITY DEVELOPMENT}

\section{Abstract}

Security is one of the most important social problems for the citizens of any country, and is a fundamental element of local development. The concept of security has been linked to social control, repression, but this idea has evolved to prevent possible conflicts.

After overcoming the traditional relationship "number of employees = more security", we analyze, with the people who inhabit the territory, what are 
the elements that create this insecurity and can we develop tools that enhance the development of community?

Keywords: Citizen Security, Local Development, Situational Prevention, Community.

\section{INTRODUCCIÓN}

El marco urbano, sumado a la relación entre territorio, comunidad y la percepción de seguridad, forma una mezcla de elementos básicos, que han sido tomados como referencia, normalmente de forma individualizada, en el estudio de la prevención del delito y en un punto más concreto, con el estudio del propio delito.

El trabajo tan complejo de prevención comunitaria, se ha venido abordando desde cualquier disciplina: geografía, economía, sociología, criminología... y siempre con un trasfondo político o de "orden social".

Una comunidad que se siente insegura, no tiene porqué vivir en un territorio inseguro. Es decir, podemos hacer una diferenciación entre objetividad y subjetividad relacionada con la percepción del entorno. La seguridad objetiva -Ser-, es aquella que maneja cifras, es la parte cuantitativa: $\mathrm{n}^{\circ}$ de delitos y faltas, detenciones, juicios... y la (in) seguridad subjetiva -Sentir- es la que los ciudadanos percibimos, no necesariamente relacionada con tipologías delictivas propias (robos, hurtos, allanamientos, homicidios...) sino simplemente, partiendo del concepto "deterioro de la ciudad como espacio de convivencia comunitaria".

Esta relación se puede comprobar a lo largo de la historia a nivel internacional, nacional o local. Podemos encontrar varios ejemplos donde se destaca el papel preventivo de la convivencia y de la comunidad como herramientas para evitar la degradación del territorio y por consecuencia, la prevención de "no lugares" y su evolución a lugares vacíos con graves problemáticas sociales.

\section{Del control estatal a la seguridad ciudadana}

Las sociedades occidentales se han basado en los sistemas de control, formales e informales, a la hora de buscar medios represivos estatales hacia conductas antisociales. Dejaron, en manos de los sistemas de control formal - Cuerpos y Fuerzas de Seguridad del Estado y al Sistema de Justicia Penal, principalmentela misión de contrarrestar las conductas antisociales que durante muchos años, según el positivismo sociológico, relacionaban directamente estas conductas con la pobreza.

Los autores de la Escuela de Chicago incluyeron en sus teorías una nueva variable, el entorno donde las personas se movían. No quiere decir que el territorio o la comunidad fuera el único elemento a tener en cuenta en la "des- 
organización social"; las desigualdades sociales, la economía, la educación y los elementos exógenos a un territorio son fundamentales en este sentido. En la actualidad, la importancia del entorno físico, es uno de los elementos más importantes que se tienen en cuenta a la hora de trabajar la prevención social; el deterioro físico del espacio ayuda a difundir una sensación, una imagen de degradación y de abandono, lo que ayuda a proponer alternativas diferentes a los medios de control o represores tradicionales.

La sociedad actual está acostumbrada a vivir en la cultura del miedo, aquella donde se nos dice que todo puede salir mal o que cualquier circunstancia puede hacernos daño convirtiéndonos en víctimas potenciales; miedo, potenciado por medios de comunicación y sustentadas por el negocio de la seguridad privada. Si nos centramos en nuestro país y en nuestra historia más cercana podemos ilustrar nuestro sistema de control formal con las palabras de B. CARLEN "La generación que alguna vez temió un Estado policial hoy presiona por un policía en cada esquina”, y es que, la respuesta al delito venía siendo el aumento de efectivos en los agentes de seguridad, mediadas de control -ej. Video-vigilancia- y el refuerzo de la seguridad privada.

El control, "la delincuencia O", es muy complicada de conseguir. Pero se pueden trabajar alternativas sociales en territorios acotados por una identidad o unas características propias comunitarias trabajando en el concepto de "eficacia colectiva" (SAMPSON, 2008). No es fácil el proceso que nos lleva desde el control estatal sobre las personas, hasta las acciones comunitarias preventivas, pero poco a poco se van importando ejemplos e iniciativas que de una forma $u$ otras abren el camino de la Seguridad ciudadana comunitaria.

\subsection{Experiencias del cambio}

Conceptos y realidades que quedan en un segundo plano en la realidad ciudadana, y en un margen con coto propio en las políticas públicas de seguridad. Encontramos, eso sí, ejemplos concretos de actividades, iniciativas, procesos y proyectos que se han llevado a cabo en nuestro entorno más cercano. Entre los países que podemos destacar como pioneros en esta idea de desarrollo y su complemento con las políticas públicas de seguridad, están el Reino Unido, Países Bajos y en un papel más secundario, a Francia. Diferentes realidades sociales y diferente problemática:

- Francia: Su problemática relacionada con el espacio urbano, su crecimiento y su falta de políticas comunitarias y de integración social han llevado a trabajar el caso y presentar informes oficiales ${ }^{1}$ como el $\mathrm{Habi-}$ liter cohabiter. Le securité dans le logement social.

1 http://www.ladocumentationfrancaise.fr/rapports-publics/024000101-habiter-cohabiter-lasecurite-dans-le-logement-social última visita 11/04/2013 
- Reino Unido: 1989, Secured by Design ${ }^{2}$, es el primer intento de difusión de los principios de prevención del delito empleando el diseño medioambiental, se instauró, por la iniciativa de la policía con el apoyo de la Home Office (Ministerio del Interior en Reino Unido)

- Holanda: Tras unos proyectos pilotos en la región de Hollans Miden, en 1996 se introduce en todo el territorio holandés, la certificación policial Politiekeurmerk Veilig Wonen ${ }^{3}$. Certificación que otorga la policía a las viviendas e instalaciones que pasan una evaluación conforme a un manual de certificación basado en los principios de prevención del delito mediante el diseño medioambiental.

Proyectos de gran envergadura, enfocados a mejorar ciertos aspectos urbanísticos, centrados, en general, en disminuir las cifras oficiales relacionadas con la comisión de delitos en nuevas zonas urbanas o en zonas ya degradadas, nunca en zonas intermedias o en proceso de envejecimiento -social, económico y ambiental-. Medidas que se introducen a la realidad social desde los gestores políticos, y que se limitan en la mejora urbanística y de seguridad, pero sin contar con la ciudadanía o con un análisis participativo en la mejora de los problemas sociales en zonas nuevas, en proceso de degradación o ya degradadas.

También tenemos ejemplos de buenas prácticas ${ }^{4}$ relacionadas con la regeneración de las zonas urbanas degradadas (crisis, paro, altos niveles de inmigración, cambios sociales...) donde los ciudadanos y ciudadanas son esenciales a la hora de poner en práctica proyectos colaborativos con las fuerzas de seguridad, servicios sociales y políticas locales de desarrollo positivo -ej: Ordenanzas de Civismo-.

Las ciudades del Foro Europeo para la Seguridad (FESU), ${ }^{5}$ son otro ejemplo de cómo la teoría pasa a la práctica. Se inspira en la argumentación que el alcalde de Bonnemaison envió al Primer Ministro Francés en el año 1982, titulado "Frente a la delincuencia: prevención, represión y solidaridad". Se habla y se defiende de la actividad desde lo local, y se promueven "las coaliciones" entre los actores sociales, que se crean tras el consenso de objetivos comunes y específicos de actuación.

Es decir, trabajar para mejorar el sentimiento de inseguridad percibida, no está relacionado la fácil solución de aumentar el número de agentes de los cuerpos de seguridad (Nacionales, de la Comunidades, locales e incluso privada) o de poner castigos más duros para aquellos que delinquen o comenten faltas administrativas; se trata de trabajar desde la comunidad para mejorar el territorio y prevenir tanto el endurecimiento de castigos, como el aumento de

2 http://www.securedbydesign.com/ Última visita 24/04/2013

3 http://www.politiekeurmerk.nl/ Última visita 03/04/2013

4 http://urbact.eu/securcity Última visita 04/02/2013

5 http://efus.eu/es/ Última visita 13/04/2013 
agentes de la autoridad pero sobre todo, que la zona se degrade tanto que se la parte cuantitativa -los delitos- focalicen el problema a resolver.

\section{SEguridAd CiUdADANA Y DESARROLLO}

El desarrollo de lo local tiene 3 vertientes, la económica, la social y la territorial. En los tres ejes hay un conjunto de actores sociales que están obligados a interactuar en busca del bien común. Desde las asociaciones vecinales, comerciales, la administración pública, políticos... y un largo etc, actores que normalmente buscan el cumplir con su misión social encomendada y olvidan el fomentar las sinergias.

Tan variado equipo comunitario, en el campo de la seguridad ciudadana, tendrá que sentarse a analizar y diagnosticar las diferentes percepciones que tienen cada una de ellas. Todas las realidades personales no son idénticas, para el colectivo vecinal una calle oscura de noche puede ser una señal de inseguridad, para un político puede ser una medida de ahorro y para el colectivo comerciante perder 3 horas de trabajo al día; distintas percepciones de una misma situación. No debemos de olvidar, que los colectivos están formados por individuos con sus propias características, y que existen realidades personales muy complejas; una víctima de violencia doméstica se sentirá más segura en la calle que en su hogar.

Trabajando con todas estas variables, debemos retomar todos los conceptos e ideas y enfocarlos, en los llamados procesos de participación ciudadana. Acotando realidades y territorios, el diagnóstico relacionado con la inseguridad ciudadana y qué medidas a seguir será más positivo. Es un proceso, no un proyecto, que debe estar representado por todos los actores a los que hemos hecho alusión en un principio, para que las medidas a adoptar sean las consensuadas y no las impuestas desde arriba, y estas medidas, deben reforzarse en iniciativas y actuaciones comunitarias más allá del incremento de la seguridad. Una mayor presencia policial y aumentar los sistemas de control y vigilancia, no aseguran un descenso de la criminalidad ni de acciones antisociales, al contrario, etiquetan a la zona de insegura y problemática.

\subsection{El turismo y el turista: necesidad de sentirse seguro}

En un primer momento, los conceptos de "Seguridad Ciudadana" y de "Desarrollo local", parecen no estar relacionados; la realidad es bien distinta, ya que podríamos afirmar que sin seguridad no encontraremos el desarrollo esperado por la comunidad; sin seguridad no hay inversión concreta: ni comercial, industrial, inmmobiliario, institucional; por tanto la zona se va degradando y se va convirtiendo en una zona muerta. Parece una conclusión bastante abstracta, pero podemos ilustrarla con un ejemplo real y práctico, es un escenario muy ligado al Desarrollo Local: El Turismo. 
Viajemos hasta Barcelona, referente turísticos a nivel internacional de nuestro país. Con identidad propia, Barcelona tiene oferta, imagen, gastronomía, infraestructuras... lo tiene todo. Uno de sus mercados más potentes entre el 2009 y el 2012 fue Japón. Según Turismo de Barcelona, en sus datos del último año -2012- el gasto del viajero japonés en la capital catalana (solo en tarjeta de crédito) alcanzó los casi 36 millones de euros. Aún hay más: Barcelona se ha posicionado como el cuarto destino europeo con un número mayor de pernoctaciones de esta nacionalidad, sólo le superan París, Londres y Florencia. Datos muy positivos para la ciudad, a los que hay que contrarrestar una situación a la que se podría calificar de de "anti place-branding" bastante perjudicial para los intereses turísticos de Barcelona, de su imagen y por contagio, al resto del territorio turístico nacional: En 2010, un 30\% de los turistas japoneses fueron víctimas de robo/hurto ${ }^{6}$. La respuesta desde el país nipón no se hizo esperar, y desde las instituciones del país, se redactó un folleto informativo sobre la peligrosidad de los territorios turísticos en España, y en concreto, de Barcelona.

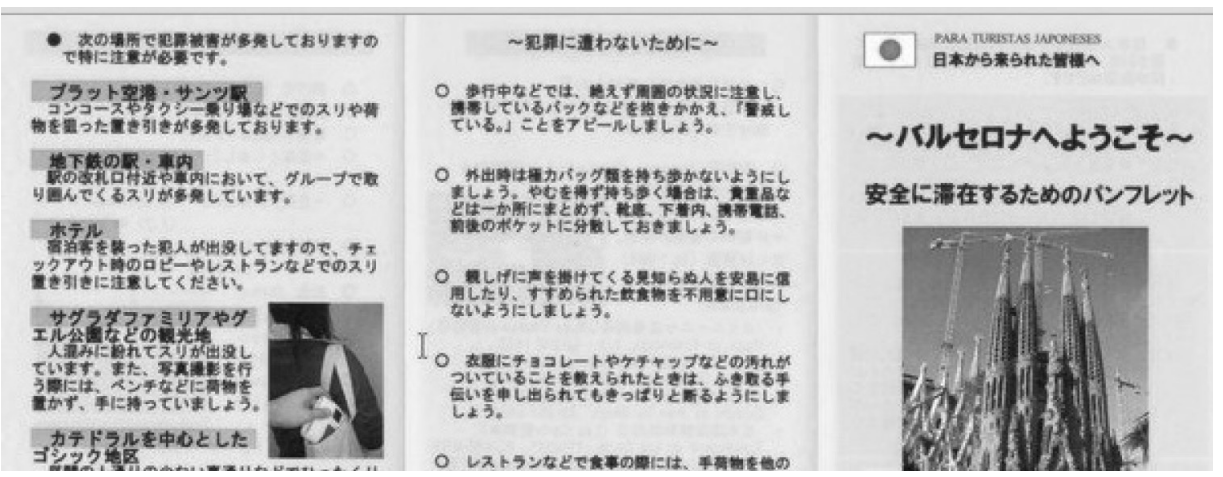

Figura 1: "Anti Place-Branding” Folleto preventivo japonés

Fuente: Ministerio del Interior.

Dependiendo de la persona que lea el folleto, nacerá una respuesta subjetiva relacionada con las sensaciones o las percepciones del individuo, y la reacción que más se identifica con la inseguridad es el miedo al delito. No es un tema baladí, ni secundario, es un tema que hay que trabajar desde "ya" si no queremos que poco a poco el sector turístico sufra las consecuencias. Tenemos un ejemplo claro y que sucedió de forma radical, el de la Primavera Árabe en Egipto y la caída del sector turístico; los turistas eran aconsejados a no viajar a las zonas afectadas por prevención. Un problema de inseguridad y un antiplace branding exterior muy bien publicitado.

6 "Alarma entre los turistas" en El Periodico.com, 13 de Octubre 2010. 


\section{LA SEGURIDAD CIUDADANA EN EL MUNICIPIO DE AliCANTE}

Alicante, es una de las ciudades españolas con una problemática acentuada en materia de seguridad ciudadana. Según el informe Deloitte donde se diagnostica a la ciudad: Los ciudadanos se sienten inseguros. Buscando datos concretos sobre la seguridad ciudadana, los podemos obtener del informe que se realizó en el municipio en el año 1988; obsoletos y una sociedad completamente distinta, son dos variables que justifican retomar el análisis de la realidad sobre seguridad en la ciudad.

Municipio con una población de 334.678 habitantes, según los datos del INE 2012, es un territorio que posee una identidad propia, dividida por distritos y a su vez por barrios. Alicante, tiene una subdivisión territorial bastante clara, donde su división más práctica, los barrios se caracterizan por tener unas condiciones socioeconómicas propias.

Proponemos un trabajo desde lo local, desde el barrio como unidad del gran municipio alicantino. El Pla del Bon Repós, es un barrio tradicional, comercial y céntrico, que en poco tiempo y como afirman sus habitantes, ha sufrido una gran transformación demográfica, económica y social.

Trabajando en su mayor parte desde análisis cualitativo, se reconoce el valor de 4 grupos de actores fundamentales: Vecinos/As, Comerciantes, visitantes y Cuerpos y Fuerzas de Seguridad. Mediante el tejido asociativo del barrio -comerciantes y vecinos- se activa la base fundamental de este trabajo: La participación ciudadana en la identificación de elementos que crean inseguridad. Como metodología contamos con encuestas semi-estructuradas, entrevistas personales y dinámicas de grupo, estas últimas para ampliar y compartir las experiencias de los/as participantes en cuanto a inseguridad.

Enfoque diferente al trabajo Municipal de 1988, donde lo que se hizo fue un análisis de datos y estadísticas policiales y judiciales contando sólo con 6 Asociaciones de toda la ciudad para que diesen respuesta a 4 preguntas abiertas sin un análisis, ni participativo para la sociedad ni representativo para la ciudad.

Teniendo un territorio delimitado, identificados los actores y haciéndoles partícipes del proceso para mejorar la convivencia y revitalizar el entorno.

\subsection{El trabajo comunitario de El Pla}

La Asociación de Vecinos del barrio del Pla, empezó a trabajar en proyectos comunitarios que no sólo eran internos, sino que los hacía extensibles a toda la ciudadanía, vecinos y vecinas, socios o no del barrio. Poco a poco, la respuesta ciudadana ha sido positiva a las llamadas de convocatoria que se hicieron desde la AVV y en cada nueva convocatoria, la suma de actores sociales es mayor. El trabajo, de algo más de un año -la nueva Junta electa se constituyó en mayo del 2012-, está creando una red barrial, inter-barrial y a nivel municipal que en pocas ocasiones se ha producido. ¿Y qué relación tiene con la seguridad ciudadana la 
labor de una AVV? La labor en sí, no tiene una relación directa con la seguridad. El difundir un proceso participativo donde todo el mundo es bienvenido, una imagen de unidad, unos objetivos comunitarios, una meta y un espacio de interacción abierta a consenso, van creando en la zona y a quienes participan un sentimiento de pertenencia a un territorio, de pertenencia a una comunidad.

El sentirse identificado con un proceso, comunidad o proyecto hace que los participantes lo sientan propio, lo defiendan y cuiden; uno de los problemas más importantes que nos encontramos en las zonas urbanas de cualquier municipio, no sólo de Alicante; es el individualismo y la falta de red social.

Iniciativas interrelacionadas que van surgiendo con otras entidades y colectivos, y van trabajando, analizando y buscando respuesta a los problemas más evidentes de El Pla: El envejecimiento de la población, la integración social, la limpieza, los solares y los vacios urbanos.

En el campo de la seguridad ciudadana, o mejor dicho, el de la percepción de inseguridad, la AVV se conformará como mediador y/o facilitador con el resto de colectivos y entidades, intentando conseguir un diagnóstico participativo lo más concreto posible sobre las zonas más vulnerables y de mejora, para que la ciudadanía pueda sentirse un poco más segura.

La percepción en la zona de que "se está degradando", según los comerciantes, hace que deban cerrar sus tiendas antes, en los meses donde oscurece pronto. En un barrio de comercio tradicional, repercute negativamente en su vida diaria ya que si los comercios cierran antes, los vecinos y vecinas vuelven antes a sus hogares, y las cales quedan abandonadas a una hora muy temprana, perdiendo la vitalidad en las calles, y para los comerciantes, perdiendo unas horas de trabajo en unas circunstancias donde el comercio tradicional está pasando en el barrio uno de sus peores momentos.

De ahí la necesidad de descubrir las necesidades en materia de seguridad que se puedan cubrir desde el ámbito comunitario, para finalmente, elaborar un mapa de hotspots que nos sirva de base a la hora de actuar.

\section{Proyectos y procesos. Conclusiones}

La seguridad ciudadana vista desde la comunidad y no desde el control puede ser un revulsivo para la situación de muchos territorios que están en proceso de degradación social, y un sistema preventivo para evitar que muchos otros inicien este proceso; en las zonas con conflictos sociales en enfoque debe ser más intervencionista.

El proyecto que se va a iniciar en el Barrio de El Pla es una pequeña parte de un gran proceso de prevención y de integración social. El proyecto de investigación, enmarcado en el Master Universitario de Desarrollo Local e Innovación Territorial de la Universidad de Alicante, conformará parte del diagnóstico que se realizará en el barrio, junto a otras actividades e iniciativas que se están llevando a cabo y cuyos resultados se integrarán en dicho trabajo 
(Mesas debate, reuniones, actividades...). Una vez reunida toda la información y analizados todos los datos, se procederá a dar respuesta a las necesidades más importantes del barrio junto a todos los actores que han participado/participarán en el proceso, para realizar e implementar acciones concretas.

Todo este proceso, no es único y exclusivo de este territorio seleccionado para la investigación. Las actividades y las iniciativas pueden ser trasladadas a otros barrios de la ciudad conservando la idiosincrasia propia de cada uno de ellos, es decir, este proceso participativo puede ser recuperado para aplicar en otras zonas -dentro o fuera de Alicante- sin olvidar la necesidad de adaptación; realidades distintas, procesos distintos.

Una parte teorica -investigación- y una parte práctica -proyectos- que intentarán mejorar la situación comunitaria. Si este proceso se repitiera en varios barrios, se podría realizar una radiografía completa de la Seguridad ciudadana en la ciudad de Alicante más allá de pasar X encuestas a un reducido número de personas, y trabajar los problemas sociales -si los hubiera- desde la raíz, de forma preventiva y no, represiva y sancionadora.

Las relaciones de pequeños elementos de la vida de un barrio, hacen comunidad.

\section{BiBLIOGRAFÍA}

AYUNTAMIENTO DE ALICANTE (1988): Estudios municipales: La seguridad ciudadana en el Municipio de Alicante Alicante: Estudios municipales.

CARLEN, B. (1998): Insecurity complex, California Lawyer, 85.

CURBET, J. (2010): La gestión de la seguridad ciudadana, Barcelona: UOC. DELOITTE (2012): Plan de competitividad Alicante 2020, Alicante: Deloitte [126,128].

GARLAN, D. (2012): La cultura del control. Crimen y orden social en la sociedad contemporánea Barcelona: Gedisa.

GONZÁLEZ PLACENCIA, L. (2002): Ciudades Seguras V: Percepción ciudadana de la inseguridad. México: Política y derecho.

SAMPSON, R. (2008): Vecindario y comunidad, eficacia colectiva y seguridad ciudadana en ORTIZ DE URBINA; J. PONCE Convivencia ciudadana, seguridad pública y urbanismo. Diez textos fundamentales del panorama internacional. Barcelona: Fundación Democracia, Gobierno Local y Diputación de Barcelona.

SECURCITY (2006): Good practice guide, Securcity network Urb-Act: ERDF Recursos electrónicos -ordenados por aparición en el texto-:

1. http://www.ladocumentationfrancaise.fr/rapports-publics/024000101-habiter-cohabiter-la-securite-dans-le-logement-social última visita 11/04/2013.

2. http://www.politiekeurmerk.nl/ Última visita 03/04/2013.

3. http://urbact.eu/securcity Última visita 04/02/2013.

4. http://efus.eu/es/ Última visita 13/04/2013. 